\title{
Asthma diagnosis and treatment - 1023. The implementation of asthma management guideline and the obstacle factors in Korea
}

\author{
Eun-Jung Jo', Mi Yeoung Kim², Suh Young Lee², Seoung-Eun Lee ${ }^{2}$, Woo-Jung Song ${ }^{2}$, Sae-Hoon Kim', \\ Sang-Heon $\mathrm{Cho}^{2}$, Kyung-up Min ${ }^{4}$, You-Young Kim ${ }^{3}$, Yoon-Seok Chang ${ }^{\text {** }}$
}

From 2nd WAO International Scientific Conference (WISC 2012)

Hyderabad, India. 6-9 December 2012

\section{Background}

There is gap between the guideline and real practice of asthma management. The implementation of asthma management guideline is essential to reduce the gap and for the qualified standard care. We evaluated the implementation of asthma management guideline and obstacle factors to the implementation in Korean physicians.

\section{Methods}

From March to April 2012, a total of 165 physicians in primary care, secondary and tertiary hospitals were enrolled. They filled in a questionnaire about their current practice on asthma: whether they followed the management guideline and if not, what might be the obstacle factors.

\section{Results}

Thirty eight percent of the physicians were male and their mean age was $43( \pm 8)$ years old. Ninety five percent of physicians had asthma patients in their clinics. Most of them (83.2\%) knew about the asthma management guideline and $87.4 \%$ of them used the guideline on their asthma practice. Among the physicians, one hundred and twenty two $(73.9 \%)$ were primary care physicians. $65.6 \%$ of the primary care physicians answered that they practiced according to the guideline for more than half of their patients. They reported difficulties in monitoring asthma control status. Only $26.2 \%$ of the primary care physicians prescribed inhaled corticosteroid (ICS) to most of their asthma patients, and the reasons that they do not prescribe ICS that much were physicians' preference for oral medications and the concern about the possible refusal by the health insurance.

\section{Conclusions}

In primary care physicians, there was a huge gap between the management guideline and real practice. This study shows the necessity of education that ICS is the first-line choice in the treatment of asthma, and strongly suggests that the current health insurance policy should be improved for the better asthma care.

\section{Author details}

${ }^{1}$ Internal Medicine, Seoul National University Bundang Hospital, Seongnam, South Korea. ${ }^{2}$ Internal Medicine, Seoul National University Hospital, Seoul, South Korea. ${ }^{3}$ Allergy and Asthma, National Medical Center, Seoul, South Korea. ${ }^{4}$ Department of Internal Medicine, Seoul National University College of Medicine, Seoul, South Korea.

Published: 23 April 2013

\section{doi:10.1186/1939-4551-6-S1-P22}

Cite this article as: Jo et al:: Asthma diagnosis and treatment - 1023.

The implementation of asthma management guideline and the obstacle factors in Korea. World Allergy Organization Journal 2013 6(Suppl 1):P22. 